\title{
Development of Compact Pulse Height Analyzer Modules Based on FPGA for E-Learning Type Exercises on Nuclear Reactor
}

\author{
Yohei IHARA $^{1^{*}}$, Wataru KADA ${ }^{1}$, Fuminobu SATO ${ }^{1}$, Toshiyuki IIDA ${ }^{1}$, \\ Junji YAMAMOTO ${ }^{2}$, Sumasu YAMADA ${ }^{2}$, Tetsuo HORIGUCHI ${ }^{3}$, and Kengo HASHIMOTO ${ }^{3}$ \\ ${ }^{1}$ Graduate School of Engineering, Osaka University, 2-1 Yamada-oka, Suita-shi,Osaka 565-0871,Japan \\ ${ }^{2}$ Faculty of Engineering, Setsunan University, 17-8 Ikedanaka-machi, Neyagawa-shi, Osaka 572-8508, Japan \\ ${ }^{3}$ Atomic Energy Research Institute, Kinki University, 3-4-1 Kowakae, Higashiosaka-shi, Osaka 577-8502, Japan
}

\begin{abstract}
To effectively perform radiation measurements for e-Learning type exercises on a nuclear reactor, we developed new, compact and inexpensive modules for a pulse height analyzer (PHA) on the basis of a field programmable gate array (FPGA). Pulse signals from radiation detectors were digitized by a free-running ADC and their digitized data were distributed in parallel to four PHA modules in the FPGA. The digitized data were processed independently in each PHA module, in other words, each user independently set measurement conditions on his own PHA module. The new PHA modules were directly connected to the Internet via local area network and were successfully used for the neutron and gamma ray measurement for the e-Learning type exercises on the Kinki University reactor.
\end{abstract}

KEYWORDS: module-type pulse height analyzer, nuclear reactor exercise, e-Learning, remote control, Internet, FPGA

\section{Introduction}

There is a request for effective execution of an education program and fruitful exercises on a nuclear reactor ${ }^{1-2)}$. It is, however, not easy to educate many students effectively in the use of an actual nuclear reactor because only a few training nuclear reactors are available. In recent years, various trials and practical applications with the Internet have been reported as effective education tools. We have also proposed an e-Learning system with an ion accelerator ${ }^{3-4}$. Nowadays, in an e-Learning on nuclear engineering, it seems to become important to execute exercises on an actual nuclear reactor.

Many techniques and methods for the digital spectroscopy have been proposed in the last decade with the development of programmable logic devices, microprocessors, analog to digital converters (ADCs), and other devices. And high-speed and high-resolution spectroscopy systems have been realized by means of field programmable gate arrays (FPGAs) and high-speed $\mathrm{ADCs}^{5-7}$. These digital processing devices are very effective in the development of intelligent and convenient nuclear instrumentation systems because the systems are reconfigurable and can be optimized to meet purposes at a low cost. Using such devices, we have also developed several digital signal processing circuits $^{8-10}$.

In this study, we designed four compact PHA modules constructed in a FPGA. Users can control the PHA modules independently and can use them as major instruments for radiation measurements. This type of PHA modules should be helpful for the effective execution of e-Learning type exercises on a nuclear reactor. At first this paper describes the core electrical circuits of the PHA modules built in the FPGA and then shows results of some experiments on their characteristics, especially in their use as major tools for

*Corresponding Author, E-mail:ihara@i-beam.jp

(C) Atomic Energy Society of Japan
e-Learning type exercises on a training nuclear reactor.

\section{Method and Apparatus}

\section{E-Learning system}

Figure 1 shows a schematic drawing of an e-Learning system supporting exercises on a training nuclear reactor. Four PHA modular circuits for the radiation measurement were constructed in a FPGA. The radiation measurement circuit with the FPGA (FPGA circuit) is set near a training nuclear reactor (a local site), and it is interconnected to four personal computers (PCs) in a lecture room (a remote site) via the Internet. The characteristics of the system can be examined through the execution of remote-controlled

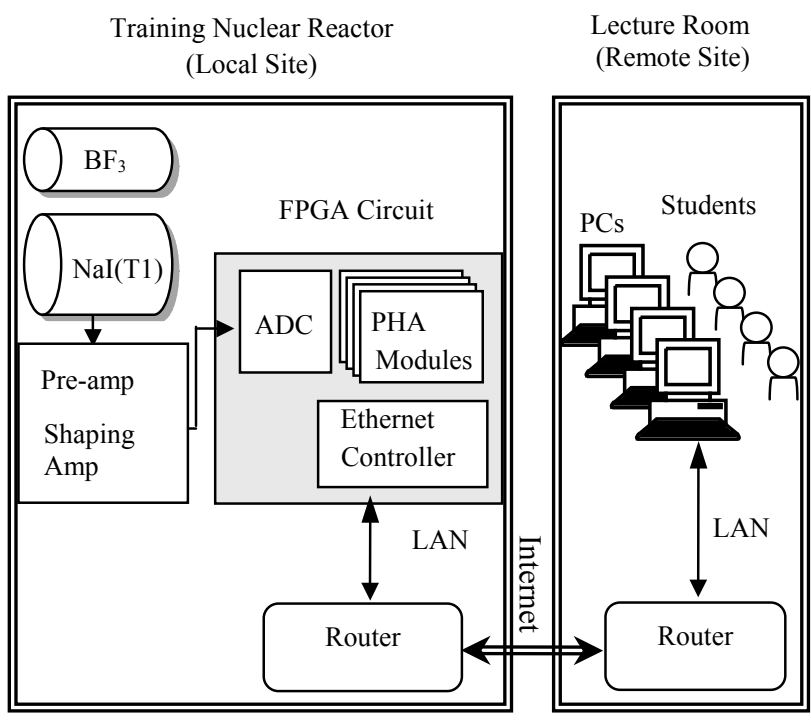

Fig. 1 Schematic drawing of e-Learning system supporting exercises on training nuclear reactor 
exercises on a training nuclear reactor. At a local site, pulse signals from a radiation detector like a $\mathrm{NaI}(\mathrm{Tl})$ scintillation counter or a $\mathrm{BF}_{3}$ proportional counter are amplified by a pre-amplifier and a shaping amplifier. The pulse-shaped signals are fed into the FPGA circuit and are in parallel processed with the four independent PHA modules. In a remote site, students have access to the PHA modules through their PCs connected to the Internet. Each student can measure neutrons or gamma-rays with each assigned PHA modules on a nuclear reactor exercise.

\section{Module-type PHA}

A schematic drawing of the FPGA circuit is shown in Fig. 2. The four PHA modules work independently in the FPGA (Cyclone EP1C3, ALTERA Corp.). Pulse signals from a $\mathrm{BF}_{3}$ or $\mathrm{NaI}(\mathrm{Tl})$ detector followed by the amplifiers are fed to a free-running ADC (AD9225, Analog Devices; 12 bits, 20MSPS) and a stream of digitized data is fed in parallel into the four PHA modules.

Two essential digital circuits were designed for the triggering and peak-detecting for input pulses. The two circuits were formed in the FPGA for the pulse height analysis of a stream of digitized data. Figure 3(a) shows a trigger circuit, which mainly consists of a comparator with 12 bits threshold voltages and two D-Flip-Flops. In case that data from the ADC exceed the threshold voltage, they are recognized as pulse signals to be analyzed. The threshold voltage is determined by each student. Figure 3(b) shows a peak detection circuit, which mainly consists of a 12 bits comparator and a 12 bits register. The output of the register is set to be the peak value of a pulse recognized by the trigger circuit. The operation of these digital circuits is synchronized with the clock $(20 \mathrm{MHz})$ for the ADC.

These trigger and peak detection circuits are effective for the saving of the resource of the FPGA. Table 1 shows the maximum number of 12 bits PHA modules, which can be formed in one FPGA. In this study, four PHA modules could be maximally formed in the FPGA. As shown in Table 1, more PHA modules can be formed in a higher grade FPGA (e.g., EP3C5). The values in Table 1 were calculated by use of the Quartus II Web Edition software (ALTERA Corp.).

Data on pulse height distributions are stored in the RAMs

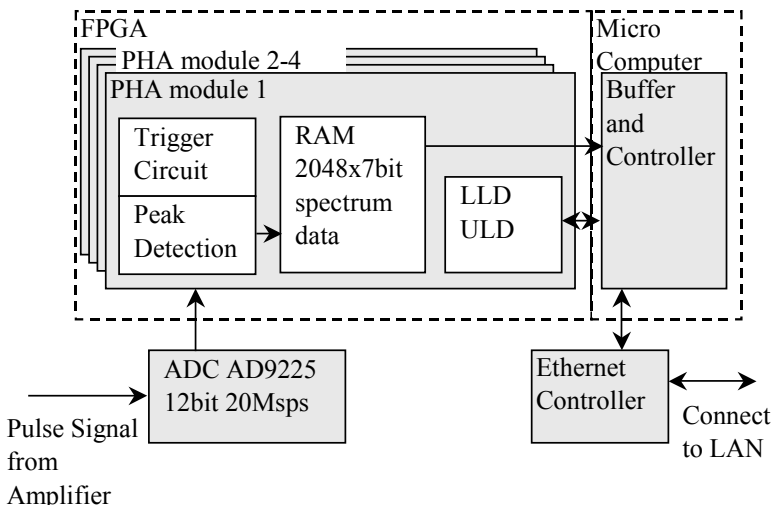

Fig. 2 Schematic drawing of FPGA circuit of the FPGA. Data in each RAM are renewed every time a pulse is detected according to the condition of each trigger circuit. Therefore, each PHA module works independently and never gives an influence to the other PHA modules. Even if a student sets a too small threshold voltage for the trigger circuit and comes to have a pulse height distribution affected with noises, other students can have suitable pulse height distributions as long as they select proper threshold voltages.

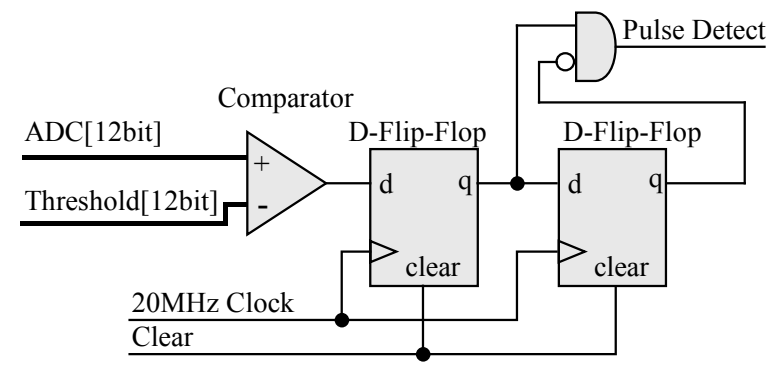

(a) Trigger circuit

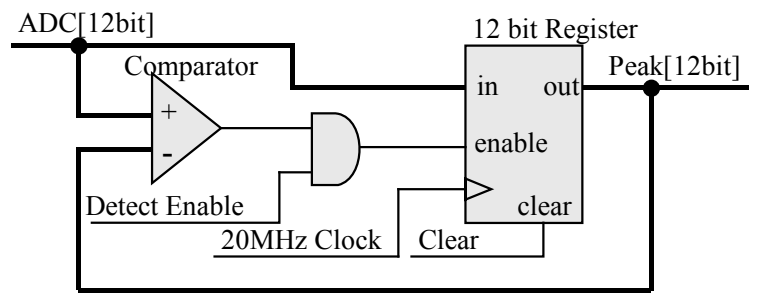

(b) Peak detection circuit

Fig. 3 Digital trigger and peak detection circuits embedded in FPGA

Table 1 The maximum number of 12 bits PHA modules, which can be formed in one FPGA. "LE" means Logic Element

\begin{tabular}{cccc}
\hline FPGA Device Name & Capacity & $\begin{array}{c}\text { The Maximum Number } \\
\text { of PHA Modules }\end{array}$ & Used \\
\hline Cyclone, EP1C3 & 2,910 LEs & 4 modules & 1,520 LEs (53\%) \\
& 60 kbit RAM & 58 kbit (94\%) \\
\hline $\begin{array}{l}\text { Cyclone, EP3C5 } \\
\text { (simulation only) }\end{array}$ & 5,136 LEs & 12 modules & 4,563 LEs (89\%) \\
\hline
\end{tabular}

\section{Network Connection and User Interfaces}

We added a web server circuit to the FPGA circuit in order to connect the system directly to the Local Area Network (LAN). A compact device server (XPort, Lantronix Inc.) was employed for the connection to the LAN. In this way, as shown in Fig.1, students in a lecture room (at a remote site) can have access to the system for the radiation measurement through the LAN and the Internet, and they can measure radiations around a nuclear reactor (at a local site). The time-sharing access is established in this system.

Figure 4 shows a view of an interface to control the PHA module, and it is controlled with a PC at a remote site via the Internet. The interface was developed by use of LabVIEW (National Instruments Corp.). There are several buttons and switches for the setting of the conditions of the PHA module 
in the left part of the page of the interface, and a view of a pulse height distribution is displayed in the right part. On the page of this interface, each student sets the conditions of each PHA module according to the requirements of the radiation measurement. Students can also save data on measured pulse height distributions in their own PCs in a file format of the comma separated value (csv). They can modify the data with their spreadsheet software.

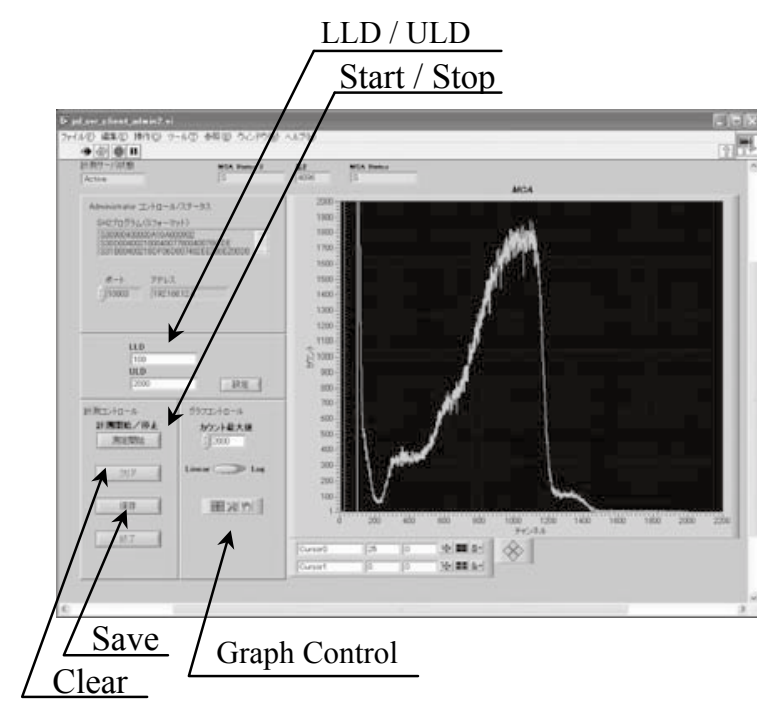

Fig. 4 Web interface to control PHA module

\section{Performance Tests and Results}

\section{Performance of PHA Modules}

Performance of the PHA modules can be roughly evaluated through the energy spectroscopy for a standard radiation source. Figure 5 shows an example of measured results of the photon energy spectrum for an ${ }^{241} \mathrm{Am}$ standard source. The measurement was performed with a Si-PIN detector (XR-100T, AMPTEK) followed by a shaping amplifier (PX2T, AMPTEK) and the present PHA modules. The position of the peaks and the values of the energy resolution for the peaks agreed well with those measured with the same detector and a commercially available multi-channel analyzer (MCA-8000A, AMPTEK). Another performance test for the PHA modules was done with a pulse generator. In the measured pulse height distribution, the full width at half maximum of the peak at a position of 1000 channel for signals from the pulse generator was less than 3 channels. It was confirmed from these performance tests that the present PHA modules could be satisfactorily used as convenient tools of the radiation spectroscopy for exercises on a nuclear reactor.

Data stored in each PHA module are sent to each PC at a remote site after the receiving of a request command from each PC via the Internet. As for the communication speed, a bottleneck is the translation speed between the microcomputer and the Ethernet controller in Fig. 2. In the present system, this bottleneck limits the total translation speed to about $230 \mathrm{kbps}$. Figure 6 shows the relation between the access rate and actual busy rate of data translation from the microcomputer to the Ethernet, and it means that no wait occurs as long as the access rate is less than 7 times per second. It should be noted that data on measured spectrum are not affected by the busy rate because the spectrum data are saved in the FPGA.

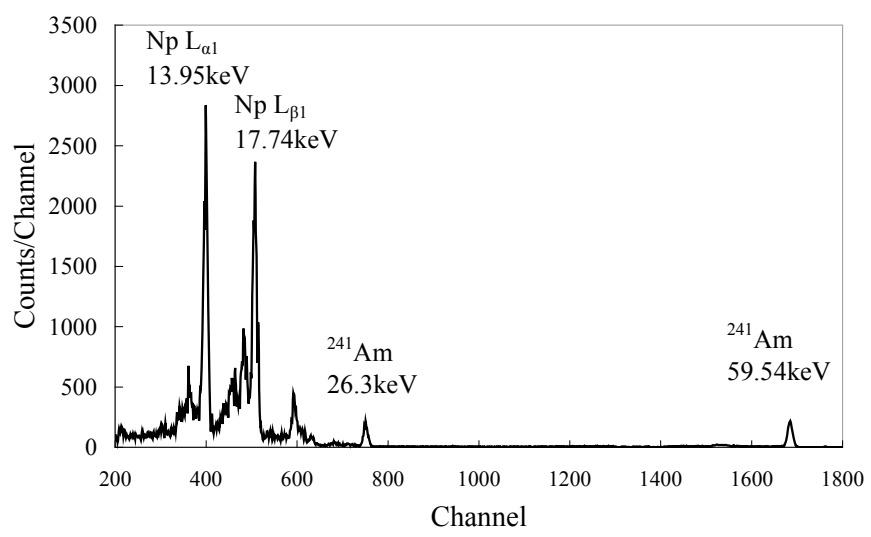

Fig. 5 Measured photon energy spectrum for ${ }^{241} \mathrm{Am}$ standard source

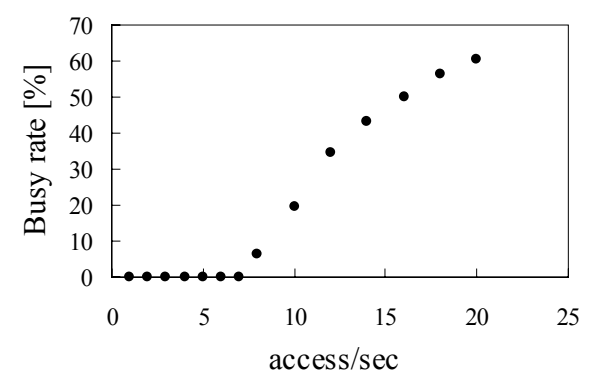

Fig. 6 Relation between access rate and actual busy rate of data translation from the microcomputer to the Ethernet

\section{Execution of E-Learning Type Exercises on Nuclear Reactor}

E-Learning type exercises on a nuclear reactor were executed by use of the present system. Four students of Osaka University (Suita city, Osaka) and four students of Setsunan University (Neyagawa city, Osaka) had the exercises. From each university, through the Internet, each student had access to each PHA module placed near the Kinki University Reactor (Higashi-osaka city, Osaka). Each student in turn set the conditions of each PHA module and acquired data on variation of neutron flux by use of a $\mathrm{BF}_{3}$ proportional counter and/or data on gamma-ray energy spectrum by use of a $\mathrm{NaI}(\mathrm{Tl})$ scintillation counter. No access error occurred for half a day of the exercises and it was confirmed that the developed compact PHA modules could be conveniently and successfully used for the neutron and gamma-ray measurement for the e-Learning type exercises on a training nuclear reactor. 


\section{Conclusion}

We developed the compact and inexpensive module-type PHA on the basis of the FPGA. In the radiation measurement system with the present PHA, pulse signals from a radiation detector followed by amplifiers are digitized by a free-running $A D C$, and then a stream of their digitized data is fed in parallel into the four PHA modules and is processed independently in each PHA module. The new PHA modules are directly connected to the Internet via LAN and they can be conveniently and successfully used for the neutron and gamma-ray measurement for the e-Learning type exercises on a training nuclear reactor.

\section{Acknowledgments}

We would like to thank Dr. Y. Ogawa of Kinki University for his technical assistance of the network operations at Kinki University. We are grateful to the staffs of Atomic Energy Research Institute of Kinki University, Setsunan University, and Osaka University for their various supports and valuable suggestions in carrying out the performance tests of the system.

\section{References}

1) M. Kitamura, Y. Tomota, S. Tanaka, et al., "Reconstruction of Nuclear Engineering Education in Universities," Nihon-Genshiryoku-Gakkai Shi (J. At. Energy Soc. Jpn.), 47[5], 311-337 (2005), [in Japanese].

2) M. Hirose, T. Tsuruta, T. Shibata, "Current status and future directions of nuclear education in elementary and secondary education,” J. Nucl. Sci. Technol., 36[3], 219-222 (1999).

3) I. Ishikawa, W. Kada, F. Sato, et al., "Development of a compact and user-friendly ion irradiation system controlled remotely through the Internet," J. Nucl. Sci. Technol., 44[8], 1039-1044 (2007).

4) I. Ishikawa, W. Kada, Y. Ihara, et al., "Construction of e-Learning system for educational program with ion accelerator controlled through the Internet," J. of JSiSE, 25[1], 98-104 (2008), [in Japanese].

5) D.K. Wehe, H. Yang, "Digital spectroscopy system for high rate events in active interrogation applications," 2007 IEEE Nucl. Sci. Symp. Conf. Rec., 1757-1761 (2007).

6) S. Buzzetti, M. Capou, C. Guazzoni, et al., "High-speed FPGA-based pulse-height analyzer for high resolution x-ray spectroscopy,” IEEE Trans. Nucl. Sci., 52[4], 854-860 (2005).

7) A. Di Odoardo, A. Geraci, G. Ripamonti, et al., "A fully digital architecture for trigger circuits in programmable logic," IEEE Trans. Nucl. Sci., 49[6], 3314-3321 (2002).

8) Y. Ihara, J. Yamamoto, S. Yamada, et al., "Embedded measurement circuit for pulse mode signal," Fall Meeting of the Atomic Energy Society of Japan, Preprints 2005, p.105 (2005), [in Japanese].

9) Y. Ihara, W. Kada, T, Iida, et al., "Development of PHA with FPGA for particle energy measurement," Annual Meeting of the Atomic Energy Society of Japan, Preprints 2006, p.414 (2006), [in Japanese].

10) Y. Ihara, A. Kishi, W. Kada, et al., "A Compact System for Measurement of Radiophotoluminescence of Phosphate Glass Dosimeter, " Radiat. Meas., 43, 542-545 (2008). 